\title{
Essais
}

Revue interdisciplinaire d'Humanités

$13 \mid 2018$

Écologie et Humanités

\section{La métaphore du viol de la terre}

Une proposition écoféministe

Noémie Moutel

\section{CpenEdition}

Journals

Édition électronique

URL : http://journals.openedition.org/essais/481

DOI : 10.4000/essais.481

ISSN : 2276-0970

Éditeur

École doctorale Montaigne Humanités

Édition imprimée

Date de publication : 1 janvier 2018

Pagination : 65-79

ISBN : 979-10-97024-03-1

ISSN : $2417-4211$

Référence électronique

Noémie Moutel, «La métaphore du viol de la terre », Essais [En ligne], 13 | 2018, mis en ligne le 01 décembre 2019, consulté le 12 décembre 2019. URL : http://journals.openedition.org/essais/481 ; DOI : 10.4000/essais.481 


\section{«Hiroi Dharti, Stri Shakti, \\ Manav Murti ${ }^{1} »$ \\ (" Terre verte, puissance féminine, \\ libération humaine ») \\ Ce que le féminisme apporte à l'écologie}

\section{Jeanne Burgart Goutal}

Loin d'être évidente, l'association entre féminisme et écologie proposée par l'" écoféminisme " peut paraître bien étrange. Quel rapport y a-t-il en effet, par exemple, entre le réchauffement climatique et les inégalités salariales entre hommes et femmes ? Entre la dégradation des ressources naturelles et l'oppression des femmes à divers degrés tout autour du globe? Cela ne va pas du tout de soi, au point que l'opinion commune, relayée par certaines féministes comme Élisabeth Badinter ${ }^{2}$, tient parfois écologie et féminisme pour des combats non seulement hétérogènes mais antagonistes, dans la mesure où l'écologie accorde une valeur centrale à la "nature ", dont le féminisme se méfie farouchement depuis Simone de Beauvoir ; ou encore, parce que " mettre en balance le rôle des objets et pratiques de la modernité dans la libération des femmes, comme les objets de consommation courante (la machine à laver) avec leur coût environnemental " risque de conduire à montrer qu' " environnement et libération des femmes ont pu (parfois) s'opposer l'un à l'autre ${ }^{3} »$. C'est pourtant de cette idée surprenante d'une connexion entre ces deux grands travers de la civilisation occidentale moderne (anti-naturalisme ou écocide d'une part ; misogynie ou patriarcat de l'autre) que part l'écoféminisme.

1 Slogan de l'organisation de femmes Mukti Sangharsh, qui lutta dans les années 1980 et1990 contre la déforestation dans l'État du Maharashtra (Inde). Cité dans Gail Omvedt, Chetna Gala et Govind Kelkar, "Unity and Struggle: A Report on Nari Mukti Sangharsh Sammelan ", Economic and Political Weekly, vol. 23, n 18, 1988 ; Mary Mellor, Feminism and Ecology, New York, New York University Press, 1997 ; Vandana Shiva (ed.), Close to Home, Londres, Earthscan, 1993, p. 5.

2 Élisabeth Badinter, Le conflit : la femme et la mère, Paris, Flammarion, 2010.

3 Lydie Cabane, note de lecture sur Une autre histoire des Trente Glorieuses, Politix, $\mathrm{n}^{\circ} 111$, 2015, p. 211. Au contraire, l'écoféminisme, issu de l'activisme féminisme antinucléaire, a dès ses débuts tenu à dissiper " l'illusion que la technologie atomique était condamnable quand elle était utilisée dans des bombes mais bienveillante quand elle était utilisée pour générer de l'électricité pour les appareils électroménagers du Nord " (Maria Mies et Vandana Shiva, Écofeminisme, Paris, L’Harmattan, 1998, p. 29). 
On pourrait questionner la pertinence d'une telle alliance du point de vue du féminisme ${ }^{4}$, mais ici, c'est sous l'angle de l'écologie que je souhaite l'interroger. Qu'apporte l'écoféminisme à la réflexion écologiste ? En quoi les concepts et points de vue développés par le féminisme, a priori sans lien avec les problèmes environnementaux, permettraient-ils en fait de féconder la pensée écologiste?

Mouvement d'actions autant que d'idées, l'écoféminisme n'est pas un courant unifié autour d'une doctrine univoque, mais une nébuleuse en constante évolution depuis son apparition voilà quarante ans, dont les différentes tendances (schématiquement : essentialiste vs critique ou même queer; matérialiste $v s$ spiritualiste) sont polarisées autour de désaccords nets, quoique cordiaux et souvent relativisés par un souci d'unité dans la lutte. L'écoféminisme a pris naissance sous forme de luttes populaires spontanées (grassroots campaigns) dans le monde anglo-saxon et le "Tiers-Monde " : dès la fin des années 1970, on voit se multiplier les mobilisations de féministes contre le nucléaire et la pollution en Grande-Bretagne et aux ÉtatsUnis. Au Kenya, en Inde, des associations de femmes se mobilisent contre la déforestation et plus largement la globalisation néolibérale et les politiques de "développement", nocives aussi bien aux femmes qu'à l'environnement. S'ensuivent les premiers manifestes et anthologies, puis des ouvrages plus académiques explorant les pistes ouvertes par l'intuition initiale de l'écoféminisme, sous des angles variés : histoire, philosophie, sociologie, théologie... Après un recul dans les années 2000, activisme et théorisation écoféministes connaissent aujourd'hui un renouveau, s'étendant notamment en Amérique du Sud (contre l'agro-industrie et l'extractivisme minier) et en Europe, où les collectifs se réclamant de cette mouvance se multiplient.

Les écoféministes articulent souci écologiste et souci féministe, à partir de la conviction qu'il existe des liens, aussi bien matériels que conceptuels, entre domination des femmes et de la nature. Écologie et féminisme, pour être conséquents et efficaces, devraient nécessairement aller de pair, voire ne seraient au fond qu'un seul et même combat. Les écoféministes entendent ainsi redonner au féminisme son ambition utopiste et révolutionnaire, réduite par le féminisme dominant (libéral et individualiste) : il ne s'agit pas seulement de militer pour l'émancipation des femmes mais pour une "mutation ${ }^{5}$ " globale de l'humanité, des rapports entre les sexes, les classes, les peuples et avec la nature, afin de résoudre ce qu'elles analysent comme une crise systémique de notre civilisation, " matrice de dominations » multiples et interconnectées, fondée sur la " mise-à-distance ${ }^{6}$ ", le dualisme et la hiérarchie.

4 Voir Jeanne Burgart Goutal, «L'écoféminisme, régression ou révolution ? », in Karine Bergès et Alexandrine Guyard-Nedelec (éds), Féminismes du XXIe siècle, Rennes, PUR, 2017.

5 Françoise d'Eaubonne, Féminisme, écologie : révolution ou mutation?, Paris, ATP, 1978.

6 Starhawk, Rêver l'obscur. Femmes, magie et politique, Paris, Cambourakis, 2015. 
Envisagé du point de vue de l'écoféminisme, le champ de la pensée écologiste actuelle apparaît comme l'emboîtement de cercles concentriques. Le premier cercle correspondrait au courant de la justice environnementale, héritier de l'écologie politique, qui croise préoccupations écologistes et sociales, travaillant à leurs intersections ; en première analyse, l'écoféminisme pourrait se présenter comme l'une de ses déclinaisons, mais je tenterai de montrer qu'il est plus que cela. Un peu plus éloigné, le cercle de l'écologie profonde (deep ecology) : comme l'écoféminisme, il remet intensément en question notre rapport à la nature en critiquant l'anthropocentrisme et le dualisme, mais sous un angle éthique qui le met à distance de l'écoféminisme, foncièrement politique. Très éloigné de l'écoféminisme enfin (au point qu'ils sont sans doute incompatibles), ce qu'on appelle parfois l'écologie superficielle (shallow ecology), le courant " modéré " de l'écologie, le plus répandu, cherche non pas à changer de système, mais à le réformer à la marge pour le "verdir ", rendre le développement plus « durable» et la croissance plus «soutenable».

À l'image des ondes se propageant autour d'une pierre lancée dans l'eau, je suivrai ces trois cercles. Je voudrais montrer que, loin de n'être qu'un cas particulier de la pensée écologiste, l'écoféminisme est un outil de critique et de dépassement de la façon même dont les problèmes y sont généralement posés et résolus dans chacune de ces trois sphères.

\section{L'écoféminisme face à l'injustice environnementale : du constat à la théorie}

Depuis quelques années, le thème "Genre et environnement " est à la mode. Mais quelle est la pertinence de cet angle de genre dans la réflexion sur la justice environnementale ? Et surtout, en quoi l'écoféminisme (qui loin d'associer simplement genre et environnement, en propose une articulation à visée révolutionnaire) permet-il de se prémunir contre le risque de récupération ou d'aseptisation des combats écologiste et féministe, que cette mode peut faire craindre?

Le point de départ des luttes écoféministes, passées et actuelles, est généralement le triple constat que la crise écologique ne touche pas à égalité tous les groupes sociaux ; que ceux-ci n'en sont pas responsables à égalité ; et que tous ne contribuent pas à égalité à la lutte contre cette crise. Plusieurs critères permettent d'étudier ces dissymétries, en les lisant en fonction d'un certain rapport de domination : la situation géographique (Nord/Sud), la " classe ", la " race ". Sans exclure ces autres critères mais en privilégiant celui du sexe, l'écoféminisme montre que les dégradations environnementales touchent davantage les femmes que les hommes, au Nord comme au Sud, et par là même, qu'elles renouvellent et renforcent le système de domination patriarcal. Contre l'idéologie dominante (même au sein du féminisme), qui voudrait que la situa- 
tion des femmes s'améliore dans une société à mesure que le capitalisme et la technologie, donc la destruction de l'environnement, y progressent, il révèle au contraire un lien entre dégradation de la nature et de la condition féminine.

Les changements climatiques exacerbent les inégalités de genre. La justice climatique est une question féministe ! Les catastrophes climatiques bouleversent socialement les communautés : le risque de subir des violences sexuelles est accru pour les femmes et les filles. Le patriarcat limite la mobilité des femmes et des filles, elles sont 14 fois plus susceptibles de mourir dans une catastrophe climatique. Les femmes des Suds n'ont pas un accès suffisant à l'information et aux ressources financières pour s'adapter au changement climatique. Les femmes sont partout dans le monde les plus vulnérables aux changements climatiques. Victimes du tsunami de 2004 : 2 femmes pour 1 homme. Victimes en France de la canicule de 2003 : 1,4 femmes pour 1 homme. Les femmes représentent 20 des 26 millions réfugié.e.s climatiques dans le monde, soit $80 \%{ }^{7}$.

Ces chiffres qui peuvent sembler étonnants s'expliquent en fait aisément : la crise écologique se surimpose à un rapport d'inégalité et de domination préexistant : si les femmes en sont les victimes privilégiées, c'est avant tout parce que « la pauvreté a un visage de femme $e^{8}$ " et que les dégradations environnementales atteignent de plein fouet les plus pauvres, qui n'ont pas les moyens d'échapper aux emplois dangereux pour la santé, aux quartiers proches de centrales nucléaires, de déchetteries toxiques, ou menacés par les catastrophes liées au changement climatique. C'est sans doute pourquoi, statistiquement, l'engagement écologiste est massivement féminin et l'engagement politique des femmes, massivement écologiste. Comme le proclame Karen Warren : "Nature is a feminist issue?! ".

Ainsi, la crise écologique aggrave la domination masculine tout en en renouvelant les modalités. Mais l'écoféminisme ne s'arrête pas à ce constat factuel, partagé avec le courant de la justice environnementale : son apport spécifique est d'en bâtir une élaboration théorique forte. Dans un souci constant de remonter des effets aux causes, les écoféministes envisagent la crise écologique comme la conséquence directe d'un modèle ou d'un "paradigme " aux multiples dimensions - social, économique, symbolique, etc. Poussant toujours plus loin la recherche des " racines du mal ", elles les trouvent ainsi dans ce qu'elles estiment être un nœud inextricable entre domination des femmes et domination de la nature, deux faces d'un seul et même processus par lequel s'est constituée la Modernité depuis le XVII siècle, voire la civilisation occidentale depuis l'Antiquité - en tout cas, le modèle culturel qui tend aujourd'hui à se mondialiser. Pour Vandana Shiva, "la vision du

7 https://feministesjusticeclimatique.wordpress.com/a-propos-2/.

8 PNUD, 1995, p. 4.

9 Karen Warren, Ecofeminist Philosophy: a Western Perspective on What it is and Why it Matters, Lanham, Rowman \& Littlefield Publishers, 2000, p. 1. 
monde réductionniste, la révolution industrielle et l'économie capitaliste sont les constituants philosophique, technologique et économique du même processus ${ }^{10}$ ", fondé sur "l'équation Homme > $($ Femme = Nature $)$ " (Ariel Salleh). Certaines auteures remontent plus loin dans le temps, comme Françoise d'Eaubonne, pour qui l'exploitation des femmes et de la nature se sont nouées lors de "la "grande défaite du sexe féminin" survenue 3000 ans avant J.-C. sur l'ensemble de la planète ${ }^{11}$ ».

Tout le monde, pratiquement, sait qu'aujourd'hui les deux menaces les plus immédiates sont la surpopulation et la destruction des ressources [...] mais très peu encore ont découvert que chacune des deux menaces est l'aboutissement logique d'une des deux découvertes parallèles qui ont donné le pouvoir aux hommes voici cinquante siècles : leur possibilité d'ensemencer la terre comme les femmes, et leur participation dans l'acte de la reproduction ${ }^{12}$.

Quoi qu'il en soit de ces désaccords historiques, l'idée est donc que la domination des femmes et de la nature constitue un inextricable "réseau " hégémonique. Un des concepts clés de l'écoféminisme éclaire cette théorie, le " capitalisme patriarcal " qui pose d'emblée l'interconnexion des dominations, montrant que l'économie capitaliste repose sur un ensemble de processus - privatisation des terres et des communs, séparation des sphères et lieux de travail productif et reproductif, marchandisation des biens et services, industrialisation - qui nuisent à l'environnement et aux femmes en une causalité circulaire : d'un côté « le prix de la destruction de ressources est externalisé et divisé de manière inégale entre les divers groupes économiques de la société, et ces coûts sont supportés en grande partie par les femmes ${ }^{13}$ ". Réciproquement, les femmes étant expertes en régénération des écosystèmes dans les sociétés à dominante agricole "en qualité de cultivatrices " et de " gardiennes des semences depuis des temps immémoriaux ${ }^{14}$ ", et assurant dans toutes les sociétés des activités qui constituent des modèles de durabilité (vie domestique, soin aux personnes...), la dégradation de leur rôle accélèrerait la destruction de l'environnement ${ }^{15}$.

10 Mies et Shiva, op. cit., p. 39.

11 Françoise d'Eaubonne, Le féminisme ou la mort, Paris, Pierre Horay, 1974, p. 241.

12 Ibid., p. 220-221. Pour une version intermédiaire : Val Plumwood, Environmental Culture, Londres, Routledge, 2001, p. 8 : «Il serait faux de croire que nous venons de tomber dans le désastre écologique du fait d'un accident technologique incongru [...]. Notre débâcle actuelle est le fruit d'une culture anthropocentriste et rationaliste vieille d'au moins deux millénaires. »

13 Mies et Shiva, op. cit., p. 91.

14 Ibid., p. 187 et 189.

15 Ibid., p. 185 : par ex. dans le domaine agricole, « la marginalisation des femmes et la destruction de la biodiversité vont de pair ". Voir aussi Shiva, Stayling Alive, Londres, Zed Books, 1989, p. 96 : «Historiquement, la nature et les femmes ont été les premières pourvoyeuses des produits d'une agriculture naturelle, fondée sur les flux durables de fertilisants émanant des forêts et des animaux domestiques vers les terres cultivées. [...] Le principe féminin de production alimentaire s'appuie sur les liens intimes existant entre les arbres, les animaux et 
Le capitalisme ne peut pas fonctionner sans le patriarcat, car le but de ce système, à savoir l'accumulation sans fin du capital, ne peut être atteint si des relations hommes-femmes de type patriarcal ne sont pas maintenues ou (re) créées. [...] Le patriarcat constitue le fondement le plus invisible du système capitaliste visible. ${ }^{16}$

Perçant une lumineuse trouée historique sur ces mécanismes obscurs, les écoféministes mettent en parallèle les phénomènes contemporains avec le processus d' "accumulation primitive du capital " des XVI et $\mathrm{XVII}{ }^{e}$ siècles, quand le capital s'est amassé entre les mains de la bourgeoisie européenne naissante par la dépossession des classes populaires, des peuples extra-européens et des femmes (dont les droits et le statut se dégradent alors terriblement). Dans ce processus, la " chasse aux sorcières " ou le "sexocide des sorcières " (qui eut lieu alors et non au Moyen-Âge comme on le croit souvent), aurait joué un rôle essentiel : fragiliser la lutte des classes en la parasitant par une lutte des sexes, rompre ainsi les solidarités populaires, déposséder de nombreuses femmes (notamment celles qui étaient indépendantes), et terrifier toutes les autres afin de pouvoir généraliser la régression de leurs statuts et droits coutumiers ${ }^{17}$. Il est frappant de noter que ces analyses trouvent un écho net dans les récits de militantes féministes pour la justice environnementale des pays dits " en voie de développement ». En Équateur et en Colombie,

[...] les industries extractives, quand elles pénètrent dans les territoires, déplacent et désarticulent les économies locales. [...] Ce processus instaure dans les communautés une économie productive hautement masculinisée, accentuant la division sexuelle du travail existante. [...] Les impacts sociaux et environnementaux comme la contamination des sources d'eau ou l'accroissement des maladies augmentent considérablement la tâche des femmes en termes de de travaux ménagers et de soins quotidiens. Un rapport réalisé dans le cadre de la "Rencontre latino-américaine : Femme et entreprise minière " qui eu lieu à Bogotá en octobre 2011 fait remarquer qu'apparaissent " des situations critiques touchant directement les femmes, comme le travail domestique, le commerce des personnes, le déplacement de femmes pour offrir des services sexuels [...]». Dans un contexte d'accélération de la masculinisation de l'espace, "l'extractivisme " réarticule les relations de genre et renforce les stéréotypes de masculinité hégémonique ${ }^{18}$.

les cultures, et sur le travail des femmes pour maintenir ces connexions. Traditionnellement, le travail des femmes en agriculture a été d'intégrer la forêt et les animaux dans l'agriculture. Une agriculture qui suit le modèle de la nature et est fondée sur la participation des femmes à la nature se reproduit d'elle-même et est durable, car les ressources renouvelées en interne fournissent les apports nécessaires pour les semences, l'humidité, les nutriments du sol et le contrôle des nuisibles. » Une analyse analogue est appliquée aux autres domaines traditionnellement féminins.

16 Mies, op. cit., p. 38.

17 Cette thèse est notamment développée dans Silvia Federici, Caliban et la sorcière, Genève-Paris, Entremonde, 2014 ; Françoise d'Eaubonne, Le sexocide des sorcières, Paris, L'esprit frappeur, 1999 ; Starhawk, Rêver l'obscur, op. cit.

18 http://www.pressegauche.org/spip.php?article17199. 
Bref, « ce plan de modernité capitaliste crée de nouveaux types de femmes, moins émancipées, et de nouvelles natures, moins libres. [...] Rien n’a changé dans la pensée "moderne" depuis plus de 300 ans. Malgré cela, les femmes comme la nature résistent ${ }^{19}$ !»

L'inégalité des sexes face aux problèmes environnementaux ne serait donc pas un hasard, ni la simple conséquence accidentelle de l'inégalité économique entre hommes et femmes ou de la division sexuelle du travail. Elle s'ancrerait dans une interconnexion systémique entre domination de la nature et domination patriarcale. Ce motif traverserait notre histoire de part en part : ce serait le thème fondamental dont l'économie capitaliste (patriarcale), la science moderne (masculiniste), ou encore les religions monothéistes, entre autres, seraient les variations. Autrement dit, il n'y aurait pas un lien seulement factuel, contingent entre dégradation de l'environnement et de la condition féminine, mais un lien systémique, nécessaire.

Pour penser ce lien, les écoféministes ont développé trois modèles théoriques distincts ${ }^{20}$. Premièrement, l'analogie : le sort des femmes et celui de la nature seraient pensables dans les mêmes termes - domination, oppression, exploitation, asservissement, réification, construction comme "Autre " de l'homme, etc. Deuxième modèle, la causalité : il n'existerait pas seulement une ressemblance, une analogie extérieure, mais une relation de cause à effet entre la domination des femmes et celle de la nature. L'idée consiste ici soit à affirmer que la domination des femmes est la cause de la domination de la nature, c'est-à-dire que les dualismes centraux de la pensée patriarcale et l'organisation patriarcale de la société sont la cause profonde de la destruction de l'environnement (Rosemary Radford Ruether soutient ainsi que " le concept de domination de la nature a été forgé, à l'origine, sur la domination sociale exercée par des groupes maîtres sur des groupes esclaves, dont la plus fondamentale est la relation entre hommes et femmes ${ }^{21}$ "; soit en inversant la perspective, que la domination de la nature est la cause de la domination des femmes, c'est-à-dire que c'est la phobie de la nature hors de nous (crainte des forces naturelles, haine de la vie, mépris des animaux...) et en nous (le corps, les affects, notre propre animalité...) qui génèrerait la phobie des femmes, souvent prises comme symboles de la nature (Dame Nature, la Terre Mère...) et de la naturalité de l'humain (" la femme est naturelle, c'est-à-dire abominable $\left.^{22} »\right)$. Troisième modèle enfin, le réseau (développé notamment par la

19 http://wrm.org.uy/fr/les-articles-du-bulletin-wrm/section1/pourquoi-les-femmes-luttentelles-contre-lextractivisme-et-le-changement-climatique/.

20 Sur ces trois modèles, cf. Jeanne Burgart Goutal, «Déconstruire le "carno-phallogocentrisme" : l'écoféminisme comme critique de la rationalité occidentale ", PhaenEx, 2016/1, n 11, p. 22-48.

21 Rosemary Radford Ruether, New Woman, New Earth, Boston, Beacon Press, 1995, p. 204.

22 Baudelaire, Mon cœur mis à nu (1887), Écrits intimes, Paris, Gallimard, 1930, p. 166. 
philosophe écoféministe Val Plumwood) : loin d'une simple causalité linéaire entre domination des femmes et de la nature, elles formeraient d'emblée un nœud, " un réseau complexe de dualismes interconnectés ${ }^{23}$ ", s'entre-constituant et s'entre-renforçant, d'où l'impossibilité de déterminer laquelle prime ou engendre l'autre.

À travers ces trois modèles, l'écoféminisme tend à montrer par une analyse " radicale » au sens propre du terme (qui dévoile les racines) que l'injustice environnementale, dont on commence à prendre conscience, serait ancrée dans une connexion ancienne et profonde, dans « les racines interconnectées de la misogynie et de la haine de la nature ${ }^{24}$ ».

\section{La critique de l'écologie profonde et de l'écologie superficielle}

Au-delà de cet approfondissement des analyses du courant de la « justice environnementale ", c'est surtout dans son dialogue avec les courants dominants de l'écologie, plus éloignés de lui, que l'écoféminisme révèle sa puissance polémique et critique. Par courants dominants, j'entends aussi bien l'écologie profonde (deep ecology) que l'écologie superficielle (shallow ecology) ${ }^{25}$. Pour l'écologie superficielle, ou " vert clair ", les racines de la crise écologique actuelle sont à chercher dans les dérives et abus de l'économie capitaliste et/ ou du système techno-scientifique moderne, qu'il suffirait donc de réformer ou de rectifier ponctuellement. Pour l'écologie profonde ou "vert foncé » au contraire, les racines du mal sont bien plus " profondes » et plongent justement au cœur même de la conscience occidentale, caractérisée dès l'Antiquité par l'anthropocentrisme et le divorce avec la nature. Les aménagements ponctuels ne suffisent pas : il faut une refonte totale de notre rapport à l'environnement pour mettre fin à cette "aliénation " délétère et développer une "identification avec le Tout " et un " égalitarisme biosphérique " qui se manifesteraient concrètement dans une conversion éthique et un changement radical de «style de vie ${ }^{26} »$.

L'écoféminisme rejette dos-à-dos ces deux analyses divergentes concernant les causes et les remèdes de la crise environnementale. Pour lui, l'écologie superficielle et même l'écologie profonde resteraient à la surface du problème, et surtout, elles resteraient imprégnées par certains des biais contre lesquels il lutte, notamment un biais androcentriste et un biais ethnocentriste.

23 Val Plumwood, Feminism and the Mastery of Nature, Londres, Routledge, 1993, p. 4.

24 Ynestra King, "The Ecology of Feminism and the Feminism of Ecology ", in Judith Plant (ed.), Healing the wounds, Londres, Greenprint, 1989, p. 24.

25 Arne Naess, "The Shallow and the Deep, Long-Range Ecology Movement: A Summary ", Inquiry, ${ }^{\circ} 16$, p. 95-100.

26 Termes empruntés à Arne Naess, Écologie, communauté et style de vie, Paris, Éditions MF, 2008. 
Pour comprendre ce soupçon, prenons trois thèmes qui sont au cœur de la pensée écologiste : la question démographique ; le concept de wilderness qui inspire la politique des parcs nationau ${ }^{27}$; l'idéal d'identification avec le tout qui constitue la clef de voûte de la deep ecology.

La question démographique entre rapidement en ligne de compte lorsque l'on évoque les causes et les solutions de la crise écologique, l'écologie prônant souvent la réduction des naissances comme dans l'un des premiers ouvrages écologistes, La Bombe P - P comme " population " - de Paul Ehrlich ${ }^{28}$, ou un an plus tard, dans le rapport du Club de Rome Halte à la croissance ${ }^{29}$. Ce néomalthusianisme débouche, concrètement, sur des campagnes de stérilisation forcée des femmes dans certains pays du Tiers-Monde ${ }^{30}$ ! En mettant en évidence ce phénomène, l'écoféminisme révèle les "implications racistes et sexistes ${ }^{31}$ " des textes et programmes écologistes mainstream : leur ethnocentrisme, puisque c'est la natalité dans le Tiers-Monde qui est posée comme un problème à résoudre ; leur androcentrisme, puisqu'ils voient la fécondité humaine (taxée de "féminine ") comme une réalité biologique, voire un fléau naturel lié au corps de la femelle humaine, au lieu de comprendre qu'il s'agit d'une réalité culturelle, liée à des coutumes et valeurs (concernant les normes sexuelles et genrées, les lois encadrant la contraception, l'avortement et l'infanticide, le type de mariage, les conceptions de la filiation.... $)^{32}$. Social et non biologique, le " problème " démographique appelle une réponse sociale et non pas technique : "Tout mouvement opposé au sexisme inhérent aux nouvelles technologies de reproduction doit commencer par reconnaître que la fertilité et la stérilité ne sont pas simplement des conditions biologiques et des "maladies" mais qu'elles sont socialement déterminées ${ }^{33} »$. Plus précisément, ce que montre l'écofémi-

27 Deux classiques du débat écoféminisme / écologie profonde : Marti Kheel, «Ecofeminism and Deep Ecology: Reflections on Identity and Difference ", et Michael Zimmerman, " Deep Ecology and Ecofeminism: the Emerging Dialogue ", in Diamond et Orenstein (eds.), Reweaving the World, San Francisco, Sierra Books Club, 1990.

28 Paul Ehrlich, La bombe P, Paris, Fayard, 1972.

29 Club de Rome / Janine Delaunay (éds), Halte à la croissance?, Paris, Fayard, 1972.

30 Mies et Shiva, op. cit., p. 212-214 et p. 307-328. Voir également l'Agenda 21 des femmes « For a Healthy Planet » présenté à la Conférence de Rio en 1992 : à lire en ligne sur le site adequations.org.

31 Ibid., titre du chap. XI, p. 197.

32 Ibid., p. 312 : "Dans la philosophie libérale du patriarcat capitaliste, on considère que le comportement sexuel des individus est déterminé par des lois naturelles qui s'expriment par des pulsions biologiques, où, comme dans le cas de l'intérêt personnel économique, les gens se livrent à leur recherche égotiste de plaisir. [...] On fait appel à cette même philosophie libérale pour justifier non seulement la séparation entre la sexualité et la procréation, mais aussi pour conceptualiser l'activité sexuelle et reproductive d'un individu comme une affaire purement personnelle, plutôt que l'expression d'une relation sociale, en connexion avec d'autres sphères et relations sociales, économiques et culturelles. "

33 Ibid., p. 211. 
nisme, c'est que ce n'est pas la nature, mais le patriarcat qui est responsable de ce que Beauvoir appelait " la fécondité absurde de la femelle humaine ${ }^{34}$ ", en assignant les femmes au rôle de reproductrices, en leur interdisant de contrôler elles-mêmes leur sexualité et leur pouvoir génératif, et en déresponsabilisant les hommes dans les questions de fécondation. C'est pourquoi selon l'écoféminisme, on ne peut résoudre justement la crise écologique sans abolir le patriarcat et élaborer une nouvelle " écologie de la reproduction " fondée sur la liberté reproductive des femmes et le respect des hommes à cet égard ${ }^{35}$.

Deuxième exemple : dès la fin du XIX ${ }^{\mathrm{e}}$ siècle, une mesure phare de la première association écologiste, le Sierra Club américain (1892), a été la création de grands parcs naturels (comme le Yosemite). Mettant en pratique certains idéaux d'Emerson, Thoreau ou Muir, il s'agissait de lutter contre l'invasion de la culture urbaine et industrielle, en en protégeant des espaces dits "sauvages ", livrés à la wilderness, où le citadin moderne pourrait se " ressourcer » dans un univers pur et intact. À l'instar de Sharon Doubiago qui parle d' " écomasculinisme ${ }^{36}$ ", les écoféministes déconstruisent l'imaginaire genré et androcentriste qui sous-tend cette obsession pour une nature "vierge ", souvent représentée comme un terrain de chasse - avec armes ou appareil photo - où "l'homme " pourrait exercer sa puissance virile, brimée par une civilisation moderne qui l'efféminerait ${ }^{37}$. Elles critiquent également cette approche en posant une question simple mais ravageuse : en quel sens et pour qui ces espaces sont-ils "sauvages " et intouchés ? Certainement pas pour les autochtones qui y vivent et en vivent - c'est-à-dire ceux des Amérindiens ou des Aborigènes qui n'ont pas encore été exterminés, dont cette idéologie occulte soigneusement l'existence et l'activité. Les écoféministes améri-

34 Simone de Beauvoir, Le deuxième sexe, Paris, Gallimard, 1949.

35 Mies et Shiva, op. cit., p. 324-326 : "Une perspective écoféministe ne consiste pas à considérer la reproduction isolément, mais l'observer à la lumière des relations hommes-femmes, de la division sexuelle du travail, des relations sexuelles et de la situation économique, politique et sociale globale qui toutes, pour le moment, sont influencées par l'idéologie et les pratiques patriarcales et capitalistes. C'est pourquoi une première exigence serait que les femmes retrouvent une plus grande autonomie à l'égard de leur sexualité et de leurs capacités procréatives. [...] Si les hommes et les femmes se mettent à comprendre les rapports sexuels comme l'interaction attentionnée et aimante avec la nature, la leur et celle de leur partenaire, ils seront capables de trouver aussi des méthodes de contrôle des naissances qui ne soient pas nuisibles pour les femmes. Cette relation faite d'amour et d'attention entraînerait une nouvelle compréhension de la sexualité. [...] Les femmes, en particulier, ont toujours connu des méthodes et des techniques de contrôle des naissances et de contraception. Une nouvelle écologie de la reproduction dans le contexte d'éco-régions économiques et politiques conduira à leur redécouverte pour assurer une densité équilibrée de gens par rapport à l'environnement sans intervention coercitive nationale ou internationale. "

36 Sharon Doubiago, "Mama Coyote talks to the boys ", in Healing the Wounds, op. cit.

37 Marti Kheel, art. cit. Pour une discussion nuancée du concept de wilderness : Ariel Salleh, Ecofeminism as Politics, NY, Zed Books, 1997, chap. 8. 
caines et australiennes révèlent ainsi que les courants habituels de l'écologie se situent implicitement à un point de vue ethnocentriste, dans le droit fil de l'imaginaire des pionniers, conquérants des Nouveaux Mondes ; elles nous incitent dès lors à renverser la focale, et à voir la question non plus de ce point de vue hégémonique, mais de points de vue marginalisés et dominés, ceux des communautés amérindiennes et aborigènes.

L'idéal présenté par la deep ecology comme la clé de voûte de la résolution de la crise écologique, la "réalisation du Soi » par "identification avec le tout ", se prête aussi au décryptage de l'écoféminisme : "Lorsque je dis que le sort de la tortue de mer ou du tigre ou du gibbon est aussi bien le mien, je pense vraiment ce que je dis. Tout ce qui est présent dans mon univers n'est pas seulement à moi, c'est moi. Et il m’appartient de me défendre moi-même $e^{38}$ ". Cette conception " exprime une théorie morale fondée sur des principes universels, c'est-à-dire sur le principe de "l'amour de l'homme" élevé à un certain degré d'impersonnalité et d'abstraction ${ }^{39}$ »; elle conduit dès lors, plus ou moins explicitement, à "l'idée selon laquelle les attachements particuliers sont moralement suspects, par opposition à une "identification" authentique, impartiale, tournant nécessairement le dos à tout ce qui est particulier ${ }^{40}$ ». Mais selon Val Plumwood, une telle dévalorisation des émotions particulières au profit de ce que l'on pourrait appeler un sentiment raisonnable abstrait (tel le " respect " chez Kant) serait conceptuellement liée à la dévalorisation des femmes et du féminin : "la sympathie, le souci, la compassion, la gratitude, l'amitié et la responsabilité [...], conçus comme la contrepartie féminine dualiste des concepts de la tradition rationaliste, et consignés dans la sphère privée comme étant de l'ordre du subjectif et de l'émotionnel, ont été tenus pour périphériques ${ }^{41}$ " de ce fait. Enfin, depuis plusieurs décennies le féminisme a construit des modèles théoriques fins pour repenser la différence et la relation (des sexes, en l'occurrence) en dépassant les "fausses alternatives " continuité / séparation. Ces modèles relationnels pourraient être appliqués de façon éclairante à la relation humain / nature (c'est ce que fait l'écoféminisme), en un " mouvement dialectique permettant de reconnaître à la fois la communauté et la différence, c'est-à-dire la mutualité $[. .$.$] , reconnaître dans la nature un autre qui est à la fois différent de soi,$ mais pas étranger ${ }^{42} »$. Pourquoi la deep ecology s'obstine-t-elle à les ignorer ?

38 Warwick Fox, Approaching Deep Ecology, Hobart, University of Tasmania, 1986, p. 60.

39 Val Plumwood "La nature, le moi et le genre : féminisme, philosophie environnementale et critique du rationalisme ", Cahiers du Genre 2015/2, n 59, p. 35.

40 Id.

41 Ibid., p. 28.

42 Val Plumwood, Feminism and the Mastery of Nature, op. cit., p. 157. Dans Ecofeminist Natures (Londres, Routledge, 1997), Noël Sturgeon recense un certain nombre des ressources conceptuelles disponibles à cet égard dans le corpus féministe anglophone : l'« identité positionnelle » 
Ces biais dissimulés derrière le traitement dominant de la question démographique, la notion de wilderness et l'idéal d'identification avec le tout, sont d'autant plus problématiques qu'ils sont précisément au cœur du paradigme " carno-phallogocentriste ${ }^{43}$ ", responsable de la crise écologique. Or comme l'écrit la black feminist Audre Lorde, "The Master's Tools Will Never Dismantle the Master's House ${ }^{44} » !$

\section{Protéger la nature ou changer le monde ? La radicalisation des idéaux écologistes}

Loin de s'arrêter à cette dimension critique, l'apport de l'écoféminisme est de repenser également les objectifs et les idéaux du combat écologiste. On formule souvent le but de l'écologie ainsi : " protéger la nature ». Mais protéger la nature ne peut être un but suffisant, ni même souhaitable, si cela passe par l'instauration d'une "dictature verte", ou par la protection d'espaces naturels et de ressources saines mais réservés à une élite au détriment de la majorité, bref par l'aggravation des inégalités face aux dégradations environnementales au sein d'un " capitalisme vert ". Les écoféministes exigent donc que l'on articule, à l'objectif environnemental, un objectif social ou politique, pensé en termes de justice, de démocratie, de meilleure répartition des ressources naturelles, des nuisances et pollutions, ainsi que du pouvoir de décision en matière environnementale, parfois aussi en termes de solidarité.

Elles soutiennent que l'égalité entre hommes et femmes, ainsi qu'entre le masculin et le féminin (c'est-à-dire la réhabilitation des activités et qualités associées aux femmes et/ou au féminin) est la condition d'une société vraiment et durablement écologique. En effet, les activités traditionnellement assignées aux femmes dans la division sexuelle du travail (agriculture de subsistance, économie domestique, soins liés au corps et à la santé quotidienne) seraient des modèles de soutenabilité écologique. Les femmes se situeraient de ce fait à un " point de vue privilégié " qui expliquerait qu'elles soient les premières à prendre conscience des problèmes environnementaux - étant chargées de procurer une nourriture saine au foyer, de soigner les enfants, les vieillards et les malades. Il faudrait donc valoriser ce savoir et en tenir compte publiquement plutôt que de le reléguer dans la sphère privée ${ }^{45}$.

d'Alcoff, l'« identité située » d'Haraway, l'« identité stratégique » de Spivak, l'« identité mobile » de Ferguson, l'« identité nomade » de Braidotti, l'« identité differentielle » de Sandoval...

43 J'emprunte ce terme à Derrida (l'une des références de Val Plumwood); voir Burgart Goutal, "Déconstruire le "carno-phallogocentrisme" ", art. cit.

44 Audre Lorde, "The Master's Tools Will Never Dismantle the Master's House », Sister Outsider, New York, Crossing Press, 1984.

45 La cinquième Déclaration de l'assemblée des femmes de la CLOC (Coordinadora Latinoamericana de Organizaciones del Campo) affirme ainsi : "C'est avec nos savoirs que 
En outre, dans la plupart des cosmologies et systèmes symboliques (en Occident ; mais aussi, quoiqu'avec des nuances et différences essentielles, en Chine : le Yin et le Yang du taoïsme ${ }^{46}$; au Japon : la Nigi-mi-tama et l'Ara-mi-tama du shintoïsme ${ }^{47}$; ou encore en Inde : la polarité Shiva / Shakti dans le tantrisme, ou Purusha / Prakriti dans la métaphysique hindouiste du Samkhya ${ }^{48}$ ), le principe féminin est associé à la fécondité, à l'unité harmonieuse, à la " force vivante qui soutient et préserve la vie ${ }^{49}$ ", autrement dit à des qualités qui devraient être au cœur d'une société pour contrebalancer nos tendances avides et prédatrices (symboliquement associées au masculin), et favoriser le soin et le respect apportés aux autres espèces, aux ressources, aux écosystèmes environnants. C'est pourquoi l'écoféminisme soutient que l'écologie doit être féministe, sans quoi elle est vouée à l'échec, et que « la planète mise au féminin reverdirait pour tous ${ }^{50}$ ».

Au-delà de cet appel à " féminiser " l'écologie, l'écoféminisme s'attache aussi à mettre en œuvre sa décolonisation. En effet, l'écologie tend généralement à aborder les problèmes et les solutions en des termes qui restent ceux du paradigme occidental, imaginant des modèles de société imprégnés par exemple de concepts d'inspiration marxiste (communisme vert, altermondialisme) ou anarchiste (autogestion, biorégionalisme...). Bref, de concepts qui, si révolutionnaires soient-ils, sont hérités de la tradition philosophique occidentale. L'écoféminisme au contraire cherche en permanence à dépasser cette manière particulière de penser qui ne serait qu'un langage hégémonique, faussement universel : il tente de mettre en ouvre un " changement de paradigme " à la fois idéel et matériel, puisant pour ce faire dans des traditions

l'agriculture a été créée. Tout au long de l'histoire, c'est nous qui avons nourri l'humanité, créé et transmis une grande partie de nos connaissances en médecine ancestrale et nous sommes actuellement celles qui produisent la plus grande partie de la nourriture, malgré les attaques contre nos terres et notre eau et tous les programmes et les politiques dirigés en permanence contre nous. Aujourd'hui, nous demandons la reconnaissance de notre contribution à la production et aux soins donnés et nous proposons de nouvelles formes de relations qui permettent de partager ces tâches et responsabilités. " Source : http://viacampesina.org/es/ index.php/temas-principales-mainmenu-27/mujeres-mainmenu-39/2382-declaracion-de-lav-asamblea-de-mujeres-de-la-cloc-la-via-campesina.

46 Dolores LaChapelle, "Sacred Land, Sacred Sex ", in Healing the Wounds, op. cit. Le Tao-te-King exhorte ainsi : "Connais en toi le masculin ; adhère au féminin. "

47 Masatsugu Maruyama, "Deconstructive Ecofeminism: a Japanese Critical Interpretation ", in Heather Eaton et Lois Ann Lorentzen (eds), Ecofeminism and Globalization, Lanham, Rowman and Littlefield Publishers Inc., 2003.

48 Vandana Shiva, Staying Alive, New Dehli, Zed Press, 1988. Elle note aussi dans Pour une désobéissance créatrice (Arles, Actes Sud, 2014) que Gandhi, inspiré par la pensée hindouiste, faisait chaque jour " deux prières. La première disait que le vrai sage est celui qui arrive à ressentir profondément et sincèrement les souffrances de l'autre et à faire acte de compassion. La seconde demandait l'aide de Dieu pour devenir plus féminin " (p. 152).

49 Shiva, Staying Alive, op. cit., p. XV.

50 Françoise d'Eaubonne, Le féminisme ou la mort, op. cit., p. 252. 
non-occidentales ${ }^{51}$. S'exposant au risque d'être taxé de folklorique, ridicule ou irrationnel, il nous exhorte à "guérir la Terre-Mère et ré-ensorceler le monde ${ }^{52}$ ", en prenant au sérieux certaines approches non-occidentales du réel qui subitement décloisonnent et font souffler un vent nouveau dans l'imaginaire politique. Les anthologies écoféministes donnent ainsi la parole non seulement à des auteures occidentales, mais aussi à des militantes de diverses communautés "indigènes » et cultures étrangères, qui tirent explicitement argument des représentations et valeurs de leur peuple $e^{53}$.

Cette décolonisation de notre imaginaire métaphysique et politique conduit par exemple Starhawk, Ruether et Shiva à décrire la Terre comme « la Déesse " primordiale, notre Mère sacrée, reprenant l'idée amérindienne de Pachamama ; elle amène Val Plumwood à qualifier les animaux non-humains et les plantes de " parents " auxquels nous devons respect et soin, " ce que veulent dire certains Aborigènes lorsqu'ils parlent de la terre comme d'une partie d'eux-mêmes, "en guise de frère et de mère" 54 "; ou encore Carolyn Merchant à penser la « justice environnementale » sous la forme d'une « éthique du partenariat ", "à l'instar de la conviction amérindienne qu'il existe un faisceau sacré de relations et d'obligations entre tous les êtres ${ }^{55}$ ".

En ce sens, l'écoféminisme radicalise et réoriente les outils conceptuels et les enjeux du combat écologiste, et tente de lui donner une portée véritablement universelle, c'est-à-dire post-coloniale donc post-occidentale.

En cohérence avec cette focale post-occidentale, l'écoféminisme fait subir aux buts et idéaux écologistes une inversion totale de perspective. Comment le courant de la justice environnementale conçoit-il en effet généralement la meilleure répartition des ressources et du pouvoir qu'il appelle de ses vœux ?

51 L'écoféminisme n'est bien sûr pas le seul courant écologiste à recourir à des traditions de pensée non occidentales; comme le montre John Baird Callicott, in Pensées de la terre. La nature dans les cultures du monde (Marseille, Wild Project, 2011), Emerson et Thoreau s'inspiraient déjà de l'hindouisme, les écologistes des années 1960 du bouddhisme zen, et ceux des années 1980 du taoïsme. Mais les écoféministes systématisent ces références, et surtout les traitent de façon réflexive et politique : il ne s'agit pas de "piller " les sagesses étrangères sur un mode néocolonialiste (risque dénoncé par Shiva, in Écoféminisme, op. cit., p. 32), mais de les mettre au cœur d'un projet altermondialiste, de prêter voix à des peuples dominés afin de leur donner $\mathrm{du}$ pouvoir dans le «système-monde » futur qu'elles militent pour faire advenir.

52 Mies et Shiva, op. cit., p. 31.

53 Par ex., dans Leonie Caldecott et Stephanie Leland (eds), Reclaim the Earth (Londres, Women's Press, 1983), la Kenyanne Wangari Maathai, la Japonaise Manami Suzuki, la Maori néozélandaise Ngahuia Te Awekotuku, l'association indienne Manushi Collective ; in Healing the wounds (op. cit.) les Indiennes Corinne Kumar D'Suza, Vandana Shiva, Pamela Philipose et Radha Bhatt, l'Amérindienne (du peuple des Gitksan West'suwet'en) Mary Wilson, l'insulaire de Haida Gwaii (archipel canadien) Gwaganad ; in Ecofeminism and Globalization (op. cit.), la Japonaise Masatsugu Maruyama et la Kenyanne Celia Nyamweru ; etc.

54 Val Plumwood, art. cit., p. 43.

55 Carolyn Merchant, "Environmentalism: From the control of nature to partnership ", 2010 : http://nature.berkeley.edu/departments/espm/env-hist/Moses.pdf. 
Comme un accès des plus démunis (pauvres, minorités ethniques, peuples du Sud, femmes...) au même statut et aux mêmes droits que les plus favorisés, autrement dit aux « privilèges des vainqueurs ${ }^{56}$ ». Vandana Shiva et Maria Mies appellent cet objectif la "stratégie de rattrapage " : il s'agirait d'une égalisation des conditions "par le haut ». Mais elles montrent qu'une telle manière de concevoir le changement est irréaliste et critiquable à plusieurs égards. Irréaliste, car elle ne tient pas suffisamment compte des limites de notre planète en matière de ressources énergétiques et biologiques, d'espèces vivantes, de capacités de régénération des sols et d'absorption des pollutions. Loin de tout prométhéisme, il s'agit de rester conscient du caractère fini de notre planète ainsi que de notre dépendance à son égard du fait de notre nature de mammiferes incarnés et ancrés (embodied and embedded ${ }^{7}$ ). En outre, la "stratégie de rattrapage " peut être critiquée pour ses biais "centristes " (le modèle à atteindre étant celui des dominants), symptômes d'une pensée hiérarchique, pyramidale : contrairement à l'écoféminisme, son but est simplement de faire accéder plus de gens au sommet de la pyramide, non pas d'abolir la structure pyramidale elle-même ${ }^{58}$.

C'est pourquoi l'écoféminisme lui substitue la " perspective de subsistance " : dans cette nouvelle optique, "la satisfaction de nos besoins basiques est à la fois le but et le chemin, pas seulement dans le Sud mais aussi dans le Nord ${ }^{59}$ "; pas seulement pour les pauvres, les indigènes, les minorités, les foyers démunis de mères célibataires, mais pour tous. Une telle conclusion "décroissante " peut sembler décevante ; mais ce que soutiennent les écoféministes, c'est que cette restriction de nos ambitions matérielles est, dans une planète finie, la condition de possibilité d'un modèle de civilisation vraiment écologiste, d'un partage réellement juste (entre humains, mais aussi entre tous les êtres vivants de la planète), et de la floraison de cultures qui expriment librement leur diversité et leur créativité - bref, du remplacement de notre vieux système-monde pyramidal par une civilisation nouvelle, celle du cercle et de "l'immanence ", où la structure même du " pouvoir-sur " serait abolie au profit du « pouvoir-du-dedans ${ }^{60} »$.

Jeanne Burgart Goutal

Université Paris-Sorbonne jeanne.burgart@yahoo.fr

56 Mies et Shiva, op. cit., p. 21.

57 Mary Mellor, Feminism and Ecology, op. cit.

58 Pour un examen de ce problème, voir Jeanne Burgart Goutal, "L'écoféminisme, une pensée de "l'identité dans la différence" ", in Monique Selim et Bernard Castelli (éds), Réparer les inégalités ?, Paris, L'Harmattan, 2016.

59 Maria Mies, "La libération des femmes et la perspective de subsistance ", in Veronika BernholdtThomsen et Maria Mies(eds), The Subsistence Perspective. Beyond Globalised Economy, Londres, Zed Books, 1999.

60 Starhawk, op. cit. 


\section{Résumé}

En quoi les concepts et points de vue développés par le féminisme, à première vue sans lien avec la crise environnementale, permettent-ils en fait de féconder la pensée écologiste ? À travers l'étude des discussions que l'écoféminisme a tissées au fil du temps avec d'autres courants, je voudrais montrer que, loin de n'être qu'un cas particulier de la pensée écologiste, il constitue un outil de critique et de dépassement de la façon même dont les problèmes y sont généralement posés.

\section{Mots-clés}

Écoféminisme, écologie, féminisme, androcentrisme, décolonial.

\section{Abstract}

In what way do the concepts and points of view developed by feminism, which at first don't seem to have anything to do with the environmental crisis, provide productive material for the ecological reflection? Through the study of discussions that emerged over time between ecofeminism and other schools of thought, I aim to show that far from being a particular instance of the ecological reflection, it is a tool to criticize and to overcome the very way problems generally are posed.

\section{Keywords}

Ecofeminism, Ecology, Feminism, Androcentrism, Decolonial. 